\title{
RACIOCÍNIO DEDUTIVO E LÓGICA MENTAL
}

\author{
DEDUCTIVE REASONING AND MENTAL LOGIC
}

\author{
Edilaine Lins GOUVEIA ${ }^{1}$ \\ Antonio ROAZZI \\ David P. O'BRIEN ${ }^{3}$ \\ Karina MOUTINHO \\ Maria da Graça B. B. DIAS 5
}

\begin{abstract}
RESUMO
Nos últimos anos, tem havido muito debate acerca da existência ou não de uma lógica mental. Essa idéia tem sofrido inúmeros ataques, tanto por estudiosos que acreditam que todo raciocínio decorre de modelos mentais (e.g., Johnson-Laird \& Byrne, 1993), como por aqueles que defendem que o raciocínio humano é dependente do conteúdo (Holyoak \& Cheng, 1995). Essa controvérsia invadiu revistas internacionais como Psychological Review, Behavioral and Brain Sciences. No entanto, os proponentes da Teoria da Lógica Mental - TLM - crêem que poucos cientistas cognitivos realmente compreendem esta teoria (O'Brien, 1998a). Diante desse quadro, o presente artigo se propõe a trazer essa discussão para o cenário nacional. Serão apresentadas sumariamente algumas teorias sobre o raciocínio dedutivo. A seguir, as principais críticas à existência de uma lógica mental; e a "defesa" dos que proclamam a existência desse tipo de lógica. Por fim, a TLM será discutida mais detalhadamente.
\end{abstract}

Palavras-chave: Raciocínio lógico, Teoria da Lógica Mental, Modelos Mentais, Teoria dos Esquemas Pragmáticos.

\section{ABSTRACT}

Nowadays, it has been having a lot of debate concerning the existence of a mental logic. This idea has been suffering countless attacks, as much for

\footnotetext{
(1) Universidade Salgado de Oliveira Endereço para correspondência: R: Dr. Gustavo Pinto, 535 - Estância Recife-Pernambuco Cep:50781-740 - Recife/PE - E-mail: edilainegouveia@ig.com.br

(2) Universidade Federal de Pernambuco - E-mail: roazzi@ufpe.br

(3) City University of New York. E-mail: dobrien@newton.baruch.eunv.edu

(4) Universidade Salgado de Oliveira.

(5) Universidade Federal de Pernambuco. E-mail: mdias@ufpe.br
} 


\begin{abstract}
specialists that believe that whole reasoning elapses of mental models (e.g., Johnson-Laird \& Byrne, 1993), as for those who defends that the human reasoning is content dependent (Holyoak \& Cheng, 1995). That controversy invaded international magazines like Psychological Review. However, the authors of the Theory of the Mental Logic - TLM - believe that few cognitive scientists really understand this theory (O'Brien, 1998a). In this context, the present article intends to bring this discussion for the national scenery. Some theories about the deductive reasoning will be presented, as well as the main critics to the existence of a mental logic and the "defense "of those who advocates in favor of the existence of this logic. Finally, TLM will be discussed.
\end{abstract}

Key words: Logical reasoning, Theory of the Mental Logic, Mental Models, Theory of Pragmatic Schemas.

\section{RACIOCÍNIO DEDUTIVO: DIVERSIDADE DE ABORDAGENS TEÓRICAS}

Vários cientistas têm se dedicado ao estudo do raciocínio dedutivo e elaborado diferentes teorias que tentam explicá-lo. De um lado, estão os que defendem a existência de uma lógica mental inerente ao raciocínio humano (e.g., Braine, 1978; O'Brien, 1995; O'Brien, Braine \& Yang, 1994; Braine \& O'Brien, 1998a; 1998b). Eles argumentam que a lógica mental desempenha papel fundamental na integração de informações e realização de inferências, pois as pessoas estão freqüentemente em contato com informações advindas de diferentes fontes e necessitam de uma lógica mental para integrar essas informações, conectá-las com informações anteriormente obtidas e realizar inferências que vão além dessas informações (Braine \& O'Brien, 1998a).

Para esses autores, a lógica mental se refere a como as pessoas combinam as proposições e raciocinam a partir das proposições compostas. Pois, ao passo que cada proposição pode ser diretamente observada, formar uma composição de proposições requer uma inferência. Assim, por exemplo, pode-se observar que "o lápis caiu no chão" e que "a ponta do lápis quebrou". E, a partir disso, concluir que "se o lápis cai no chão, então sua ponta quebra". Entretanto, a relação condicional não pode ser diretamente observada. Essas conexões são inferidas. Os procedimentos de inferência têm que preservar os valores-verdade das proposições. Assim, a lógica mental trata da realização de inferências proposicionais. Para os proponentes da TLM, o raciocínio cotidiano utiliza procedimentos perfeitos de inferências por meio dos quais, partindo-se de proposições supostamente verdadeiras, inferem-se proposições que herdam este valor-verdade. Ou seja, inerente ao raciocínio humano, existe um sistema de dedução natural.

De outro lado, estão os que advogam contra a existência desse tipo de lógica. JohnsonLaird, Byrne e Shaeken (1992), por exemplo, afirmam que as pessoas não usam esquemas de inferência. Em vez disso, elas constroem representações internas, denominadas modelos mentais, e realizam as inferências a partir desses modelos. Para esses autores, o raciocínio dedutivo depende de três processos principais: construir um conjunto de modelos mentais a partir da informação inicial para o raciocínio conjuntamente com qualquer conhecimento prévio relevante; tentar formular uma conclusão provisória a partir de um modelo mental; e, por fim, verificar a validade da conclusão, certificandose de que nenhum modelo alternativo, partindo das mesmas premissas, torna a conclusão provisória falsa. Se não houver nenhum modelo mental que a falsifique, a conclusão é considerada válida. O raciocínio consiste, assim, em buscar modelos mentais alternativos que poderiam falsificar a conclusão que se está analisando. Ou seja, baseia-se em processos semânticos, e não sintáticos. 
Para Cheng e Holyoak (1985), os processos subjacentes ao raciocínio dedutivo não são sintáticos, isto é, baseados em regras formais, nem semânticos - por meio da manipulação de modelos mentais. Eles argumentam que as pessoas são capazes de raciocinar corretamente a respeito de assuntos concretos, realísticos ou temáticos por meio de estruturas de conhecimento abstratas aprendidas indutivamente, tais como "permissão" e "obrigação". Essas estruturas, denominadas esquemas de raciocínio pragmáticos, consistem em conjuntos de regras generalizadas e dependentes do contexto que, diferentemente de regras puramente sintáticas, são definidas em termos de classes de objetivos (tais como realizar uma ação desejada) e relações entre esses objetivos (cumprir um pré-requisito e poder realizar a ação desejada). Eles defendem que as pessoas, ao raciocinar, utilizam exclusivamente esquemas de raciocínio pragmáticos.

Percebe-se, assim, que os estudiosos que tentam compreender e explicar esse tipo de raciocínio não conseguem chegar a um consenso sobre seus processos subjacentes. Esta é uma área de estudo em que ainda há muito a ser "desvendado". A controvérsia com relação à existência ou não de uma lógica mental tem, nos últimos 10 anos, sido tema de consideráveis debates entre esses cientistas, invadindo revistas como Psychological Review, Behavioral and Brain Sciences, Cognition e o Journal of Experimental Psychology: General. No entanto, os proponentes da Teoria da Lógica Mental - TLM - crêem que poucos cientistas cognitivos realmente compreendem o que é esta teoria e quais as suas afirmações (O'Brien, 1998a). As principais questões inseridas nesse debate serão apresentadas a seguir.

\section{ARGUMENTOS CONTRÁRIOS À EXISTÊNCIA DE UMA LÓGICA MENTAL}

Os teóricos contrários à existência de uma lógica mental fundamentam seus argumentos no fato de que a maioria das pessoas falha ao tentar resolver problemas lógicos. Dentre estes problemas, a tarefa de seleção de Wason (Wason, 1966; Wason \& Johnson-Laird, 1972) tem sido a mais estudada. Nesta tarefa, apresenta-se ao sujeito quatro cartas. Cada carta possui um número (par ou ímpar) em um lado e uma letra (vogal ou consonante) no outro. O objetivo é identificar as cartas que (e apenas as que) precisam ser viradas para determinar a veracidade da seguinte regra ou hipótese: "Se existir um número ímpar em um lado de um cartão, então haverá uma vogal do outro lado". Em uma das versões originais da tarefa, as cartas apresentadas ao sujeito são "1, 2, A e B". O sujeito pode testar sua hipótese virando somente as cartas necessárias para determinar, de forma conclusiva, se a hipótese é verdadeira ou falsa para este conjunto de cartas. A regra apresentada é uma das formulações lingüísticas que correspondem à relação lógica "Se p então q" (regra condicional ou de implicação). As quatros cartas são exemplos que representam "p", "não p", "q" e "não q" (respectivamente, 1, 2, A e B nesta forma de apresentação).

Esta tarefa, na forma abstrata acima, é de difícil resolução: só 4\% de estudantes universitários selecionam a resposta correta (Wason \& Johnson-Laird, 1972). A maioria dos estudantes escolhe virar só a carta " 1 " ou as cartas "1" e "A". Provavelmente eles escolhem a carta " 1 " porque, encontrando uma letra " $A$ " do outro lado, confirmaria a hipótese e escolhem a carta " $A$ " com a idéia de que encontrar o número " 1 " do outro lado também iria confirmar a hipótese. Neste sentido, estes sujeitos escolhem somente as cartas capazes de verificar a regra. A resposta correta, entretanto, é virar só as cartas "1" e "B", as únicas cartas capazes de demonstrar a regra como falsa. Pois, diante da hipótese: "Se uma carta possui um número ímpar de um lado, então do outro lado tem uma vogal", uma carta com um número ímpar e uma vogal é, obviamente, consistente com a hipótese. Por outro lado, uma carta com um número par é consistente com a hipótese independentemente de ter uma vogal ou uma consoante do outro lado, pois a hipótese 
não faz nenhuma previsão para esta eventualidade. Assim, só uma carta com um número ímpar e uma consoante não é consistente com a hipótese. Conseqüentemente, é preciso virar a carta com o número ímpar (" $p$ ") para ver se tem uma consoante do outro lado e, ao mesmo tempo, virar a carta com a consoante ("não q") para verificar se tem um número ímpar do outro lado. Não é necessário se preocupar com as outras duas cartas - "2" ("não p") e "A" ("q") - porque nenhuma delas é capaz de demonstrar a regra como falsa conforme explicado anteriormente.

Poder-se-ia pensar que, fornecendo essas explicações sobre a resolução correta da tarefa, o desempenho dos sujeitos melhoraria. Entretanto, Wason e Johnson-Laird (1972) constataram que a dificuldade em fornecer a resposta correta é insensível às explicações, pois, após entrevistas em que as cartas foram discutidas, os sujeitos permaneceram com as mesmas dificuldades.

Em suma, a tarefa de seleção de Wason tem sido apresentada como evidência empírica bastante conspícua contra a existência de uma lógica mental visto que, além de a maioria das pessoas não conseguir resolvê-la corretamente, explicações detalhadas acerca de sua resolução praticamente não alteram o desempenho dos sujeitos.

Outro forte argumento dos teóricos contrários à existência da lógica mental é que, paralelamente ao fracasso na resolução da tarefa abstrata de Wason, pesquisas têm demonstrado que as pessoas são capazes de responder de forma logicamente apropriada a versões desta tarefa que são apresentadas com conteúdos realísticos. Um exemplo é a versão da tarefa introduzida por Griggs e Cox (1982) denominada "Drinking-Age Problem". Nesta tarefa, diz-se ao participante para imaginar que é um policial aplicando a seguinte lei: "Se a pessoa está tomando bebida alcoólica, então ela tem que ter pelo menos 21 anos de idade". Quatro cartas são apresentadas, cada uma delas com a idade da pessoa de um lado e a bebida do outro. Os sujeitos são instruídos a virar aquelas cartas, e apenas aquelas, que poderiam levar a descobrir o violador da regra. Muitas pessoas selecionam as cartas logicamente apropriadas - a da bebida alcoólica e a da pessoa com menos de 21 anos (O’Brien, 1998b).

O sucesso na resolução destes tipos de versões da tarefa de seleção tem sido freqüentemente apresentado como uma evidência a favor das teorias conteúdo-dependentes. Além de serem capazes de raciocinar corretamente diante de versões com conteúdo realísticos, Cheng e Holyoak (1985) argumentam que, de acordo com a Teoria dos Esquemas Pragmáticos, as pessoas raciocinam a partir de estruturas de conhecimento abstratas, então elas devem ser capazes de resolver problemas de seleção abstratos desde que, em sua solução, haja 'elicitação' dessas estruturas, isto é, que o problema apresente uma regra pragmática.

Eles elaboraram um experimento (Experimento 2, apresentado em 1985) constando de duas versões do problema de seleção desprovidas de conteúdos realísticos. Na versão experimental, havia uma regra pragmática de permissão. A instrução apresentada era a seguinte:

"Suponha que você é uma autoridade verificando se as pessoas estão obedecendo ou não a determinados regulamentos. Os regulamentos possuem a seguinte forma: Se uma pessoa executa a ação "A", então se deve primeiramente satisfazer a precondição " $P$ ”. Em outras palavras, para ser permitido " $A$ ", deve-se primeiramente ter satisfeito o pré-requisito "P". Os cartões abaixo contêm informações acerca de quatro pessoas. Um lado do cartão indica se uma pessoa tem executado ou não a ação "A", o outro lado indica se este mesmo indivíduo tem satisfeito a precondição "P". Para verificar se um determinado regulamento tem sido obedecido, quais cartões abaixo você viraria? Vire somente os cartões que você precisa para verificar, para ter certeza. 
Os quatro cartões mostram "executa ação A", "não tem executado a ação A", "satisfaz a precondição $P$ ", "não satisfaz a precondição P"6.

Para avaliar o papel do esquema de permissão em facilitar respostas corretas, os sujeitos eram também examinados em uma versão não-pragmática do problema de seleção - problema de controle - apresentado da seguinte forma:

"Abaixo há quatro cartões. Cada cartão possui uma letra de um lado e um número do outro lado. Sua tarefa é decidir qual dos cartões você precisa virar para verificar se uma certa regra está sendo seguida ou não. A regra é: Se um cartão possui um " $A$ " em um lado, então deve ter um 4 do outro lado. Vire apenas aqueles cartões que você precisa para ter certeza.

Os cartões mostram a letra "A", "B" (i.e., não A), "4" e "7" (i.e., não 4) 7.

Este experimento se tornou a evidência mais persuasiva a favor da teoria proposta por Cheng e Holyoak pois, conforme eles prognosticaram, o problema baseado em uma afirmação abstrata de uma regra de permissão produziu substancialmente melhores níveis de desempenhos do que um problema comparável que não apresentou este tipo de regra. Esses resultados sugerem que apenas a presença da regra pragmática - sem qualquer conteúdo familiar - seria suficiente para viabilizar as escolhas corretas.

\section{ANÁLISE MAIS DETALHADA DESSES ARGUMENTOS}

Em conjunto, os experimentos citados anteriormente demonstram que a maioria das pessoas é incapaz de resolver corretamente versões abstratas de problemas lógicos e treiná-las na resolução correta dos problemas praticamente não afeta seu desempenho, mas versões desses problemas com conteúdo realístico, bem como versões abstratas que apresentam regra pragmática, são resolvidas corretamente pela maioria das pessoas. Parece, assim, que o que viabiliza o raciocínio lógico correto é a presença do conteúdo realístico e/ou da regra pragmática. Entretanto, faz-se necessário retornar às evidências empíricas anteriormente apresentadas e analisá-las mais detalhadamente antes de se aceitar essa afirmação.

Com relação ao experimento de Cheng e Holyoak, é importante ressaltar que, do ponto de vista metodológico, os problemas deveriam diferir entre si apenas com relação à presença/ausência da regra pragmática de permissão. Entretanto, observa-se a existência de várias diferenças entre os dois problemas: (1) Presença de negativas explícitas no problema experimental ("não executa ação $A$ " e "não satisfaz a precondição $P$ ") e de negativas implícitas no problema controle - "B" em "B (i.e., não A)" e o "7" em "7 (i.e. não 4)"; (2) Presença de um contexto de verificação (i.e., "Suponha que você é uma autoridade verificando se as pessoas estão obedecendo ou não a determinados regulamentos") e de uma afirmação que clarificava a regra no problema experimental (" $E m$ outras palavras, para ser permitido " $A$ ", deve-se primeiramente ter satisfeito o pré-requisito " $P$ ") que não estavam presentes no problema de controle; (3) Nos problemas originais em inglês, observa-se maior número de palavras no problema experimental, 121, do que no problema de controle, 81; (4) As instruções do problema experimental incluíam uma questão a mais - "Para verificar se um determinado regulamento tem sido obedecido, quais cartões abaixo você viraria?"- do que a apresentada no problema controle; (5) A ordem na qual as informações foram apresentadas variou entre os problemas: no problema experimental, a regra foi apresentada antes que os conteúdos dos cartões fossem descritos e no problema controle, os cartões eram descritos antes das instruções e da regra.

(6) Tradução feita pelos autores do artigo de Cheng e Holyoak, 1985, p. 403.

(7) Tradução feita pelos autores do artigo de Cheng e Holyoak, 1985, p. 403. 
Desta forma, verifica-se que o problema experimental tem características "mais ricas" do que o problema controle e esta diferença de características confunde a variável de interesse - a presença da regra pragmática. Faz-se necessário, então, investigar quais variáveis, de fato, estão influenciando o desempenho dos sujeitos. Vários estudos têm se dedicado a esta tarefa. Jackson e Griggs (1990), por exemplo, verificaram que removendo as negativas explícitas do problema experimental, o desempenho dos sujeitos cai bastante. Em outro experimento (Experimento 3), eles constataram que o uso de negativas explícitas no problema controle, por outro lado, não resultava em respostas corretas como ocorria com o problema experimental. No Experimento 4, esses autores removeram o contexto de verificação do problema experimental e, conservando as negativas explícitas, obtiveram baixa percentagem de respostas corretas. Percebe-se, então, que as outras variáveis existentes entre os dois problemas apresentados por Cheng e Holyoak (1985) estão interferindo no desempenho dos sujeitos. Assim, não se pode atribuir o bom desempenho no problema que apresenta a regra de permissão abstrata apenas à 'elicitação' do esquema pragmático, como pretendido por esses autores.

Ainda neste sentido, Noveck e O'Brien (citados em O'Brien, 1998b) encontraram versões abstratas não-pragmáticas que, incluindo algumas das características cruciais do problema contendo a regra abstrata de permissão, são resolvidas por muitas pessoas. Parece, então, que a resolução deste tipo de problema não tem nada a ver com a natureza permissionária da regra, mas sim com essas outras características extrínsecas à regra. Diante dessas evidências, a afirmação de que a resolução de problemas envolvendo a presença da regra pragmática decorre da presença desta regra e da conseqüente 'elicitação' do esquema pragmático não se sustenta.

Voltando-se agora para a análise das versões conteúdo-realistas, é importante destacar que, para afirmar que o melhor desempenho nessas versões se deve à presença deste tipo de conteúdo, seria necessário que esta fosse a única variável existente entre os dois tipos de versões da tarefa de seleção. Entretanto, O'Brien (1998b) ressalta que há diferença estrutural entre as versões abstratas e as versões conteúdo-realistas. A tarefa abstrata de Wason é metalógica, i.e., solicita que se raciocine sobre a regra. As versões com conteúdos realistas, por outro lado, são tarefas lógicas, nas quais se raciocina a partir da regra: a regra é supostamente verdadeira e pode ser diretamente usada para se chegar à conclusão. Deste forma, não faz sentido afirmar que a presença do conteúdo realístico viabiliza a ocorrência do raciocínio lógico correto, uma vez que o bom desempenho das pessoas nestas versões parece ser devido à maior facilidade estrutural desses problemas.

As pessoas dificilmente resolvem a tarefa de seleção de Wason de forma correta. Esse fato não é contestado pelos defensores da existência de uma lógica mental. Entretanto, para eles, toda essa polêmica em torno da tarefa de Wason e suas versões não é capaz de afetar a teoria que propõem, tendo em vista que a resolução desta tarefa pela maioria das pessoas está fora das predições de sua teoria.

A este respeito, O'Brien (1995) argumenta que as pessoas raciocinam logicamente quando as demandas da tarefa estão inseridas no escopo das habilidades básicas da lógica mental. Quando as demandas excedem essas habilidades, como é o caso da tarefa de Wason, as pessoas têm que contar com outros processos e podem cometer erros e dar respostas que não mostram nenhuma evidência de qualquer lógica mental. Portanto, ele considera que a não resolução dessa tarefa não é capaz de atacar a teoria que propõe, tendo em vista que sua resolução não é proclamada por essa teoria.

Em suma, as críticas feitas à TLM não se sustentam quer seja por falhas metodológicas dos experimentos que servem de alicerce para as conclusões proclamadas pelos oponentes desta teoria, quer seja por se basearem em 
compreensões errôneas acerca do que a teoria, que será apresentada a seguir, afirma.

\section{TEORIA DA LÓGICA MENTAL (TLM)}

Essa teoria parte do pressuposto de que o raciocínio lógico é proposicional, pois a lógica se preocupa com a veracidade das conclusões. Diferentemente das proposições que têm valor-verdade definido, i.e., são verdadeiras ou falsas, as sentenças não têm definição de valorverdade. Ora elas podem ser verdadeiras ora falsas como, por exemplo, a sentença "Eu sou pernambucana" será verdadeira quando dita por uma mulher nascida em Pernambuco, mas não o será quando dita por qualquer homem ou por uma mulher não nascida neste Estado. A sentença não pode ser julgada como verdadeira ou falsa até que se saiba em que condições ela foi produzida. Assim, as sentenças estão fora do escopo da TLM, pois, para a realização de inferências lógicas, é fundamental que o sujeito acredite que as premissas são verdadeiras (proposições verdadeiras), ou seja, que ele as trate como suposições (O’Brien, 1998b).

Dentre os diversos tipos de inferência que as pessoas fazem ao raciocinar, estão as inferências lógicas, que correspondem ao interesse central da TLM. No entanto, esta teoria não afirma a exclusividade deste tipo de inferências. Ao contrário, defende que diversos tipos de inferências coexistem pacificamente no raciocínio humano, no qual os resultados de um tipo de inferência podem servir de input para outro tipo.

A TLM se propõe a descrever as inferências lógicas que são realizadas rotineiramente em uma variedade de atividades, incluindo raciocínio, conversas e compreensão de textos (e.g. Braine, 1978; Braine, Reiser, \& Rumain, 1998; Braine, O'Brien, e cols, 1998; Lea, 1998). Essas inferências dependem de uma lógica mental e são realizadas sempre que as premissas estão presentes na memória de trabalho e as pessoas estão motivadas a compreender as informações que estão processando (O'Brien, Braine \& Yang, 1994).

A TLM possui três partes: os esquemas de inferência, o programa de raciocínio e a pragmática. Os esquemas de inferência determinam qual tipo de inferência pode ser realizado a partir de tipos particulares de premissas. Eles especificam como as partículas lógicas - e, ou, se - e a negação podem ser usadas no raciocínio e definem o repertório de etapas dedutivas elementares disponíveis para os sujeitos no raciocínio proposicional (para mais detalhes sobre 0 repertório de esquemas de inferência e o programa de raciocínio, consultar Braine e O’Brien,1998c).

O programa de raciocínio, por sua vez, é um programa de dedução natural que modela como o sujeito constrói uma série de raciocínio usando os esquemas. A pessoa não tem acesso introspectivo direto a seus esquemas. O uso dos esquemas é controlado pelo programa de raciocínio, que descreve como o esquema que será aplicado em determinado ponto da série de raciocínio é selecionado e inclui rotinas de raciocínio direto, que são universais, e estratégias de raciocínio mais sofisticadas.

A parte de rotina - rotinas de raciocínio direto (RRD) - é universal e consiste em um programa simples que, diante das premissas, identifica o esquema que pode ser aplicado e o utiliza. O resultado desta aplicação é avaliado e, se necessário, adicionado ao conjunto de premissas para nova aplicação de esquema. As RRD são aplicadas rotineiramente e sem esforço tanto na compreensão de textos e de discursos (Lea e cols, citados em Lea, 1998) quanto nas tarefas de raciocínio (e.g., Braine, Reiser \& Rumain, 1998; Braine, O’Brien e cols, 1998).

Diversos estudos têm demonstrado que as inferências vinculadas às RRD são realizadas sem que as pessoas se dêem conta. Lea e cols (citados em Lea, 1998), por exemplo, verificaram que esse tipo de inferência é realizado automaticamente na compreensão de textos. Eles solicitaram aos sujeitos que lessem um texto e depois indicassem dentre três frases - uma 
contendo informações explicitamente apresentadas no texto, outra com inferência derivada das RRD e a terceira com inferências previstas pela lógica formal, mas não previstas pela TLM - aquelas que estavam presentes no texto. Verificaram que tanto as informações que foram explicitamente apresentadas quanto as inferências decorrentes das RRD foram identificadas pelas pessoas como presentes no texto. Ou seja, os sujeitos realizavam as inferências de forma tão automática e sem esforço que sequer se davam conta de estarem inferindo.

As estratégias mais sofisticadas, por sua vez, exigem alguma reflexão ou intuição para serem adquiridas. Logo, não estão universalmente disponíveis. Essas estratégias apenas entram em operação quando as RRD falham, i.e., não conseguem determinar a resposta (O’Brien, Braine \& Yang, 1994). As inferências que requerem estratégias de raciocínio indireto serão menos freqüentemente realizadas do que aquelas vinculadas às RRD.

A terceira parte da TLM se refere às influências pragmáticas sobre o raciocínio. Esta teoria propõe que o significado básico de cada partícula lógica está nas inferências que são autorizadas pelos seus esquemas. No entanto, os esquemas de inferência agem sobre as representações semânticas, que são o produto dos processos de compreensão. Vários fatores afetam a forma como a tarefa é compreendida/interpretada pelas pessoas e como as proposições são construídas por elas, tais como o conhecimento de mundo, as crenças pessoais, o tipo de conteúdo apresentado, as instruções fornecidas e até mesmo a ordem de apresentação das tarefas. Assim, as informações que servem de base para a realização de inferências são afetadas por todos os processos pragmáticos de compreensão.

A este respeito, Braine e O'Brien (1998b) apresentam três princípios gerais para a explicação da influência dos fatores pragmáticos na compreensão. O primeiro se refere à influência do conteúdo na forma como as proposições são construídas. Tem sido amplamente reconhecido na literatura (e.g., Byrne, Espino \& Santamaria, 1999; Vadeboncoeur \& Markovits, 1999; Markovits, 1988; Dias, 1996) que as pessoas se baseiam em seu conhecimento prévio para dar sentido à situação atual. Assim, interpretações que lhes parecem plausíveis, coerentes com sua visão de mundo, são mais provavelmente construídas do que outras que pareçam pouco plausíveis. Desta forma, as pessoas podem, diante de tarefas lógicas, estar se baseando em premissas diferentes daquelas propostas pelo experimentador, se elas Ihes parecerem pouco plausíveis.

O segundo princípio se refere à lógica da conversação formulada por Grice (citado em Guimarães, 1995). De acordo com este autor, há uma diferenciação entre o significado literal de um enunciado e o sentido intencional do falante ao enunciá-lo. Ele argumenta que o sentido do que o interlocutor diz deve ser interpretado levando em conta tanto o sentido literal do que foi dito, ou seja, o que o enunciado significa independentemente de sua situação de uso, quanto a situação em que é dito. O modo como a situação opera está relacionado com o que Grice chamou de implicações conversacionais

“... conversational implicatures (which) are inferences that arise on the basis of some general rules or maxims of conversational behavior..." (Levinson, 1983, p. 10)

Guimarães (1995) afirma que, segundo Grice, a conversação é regida pelo Princípio da Cooperação que informa ao falante para fazer sua contribuição conversacional tal como é requerida, no momento em que ocorre, pelo propósito ou pela direção do intercâmbio conversacional em que ele está engajado. No interior desse princípio, funciona um conjunto de regras denominadas de Máximas Conversacionais, que são as seguintes: faça com que sua contribuição seja tão informativa quanto requerida para o propósito corrente da conversação, i.e. faça com que ela não seja nem menos nem mais informativa do que requerida; não diga o que acredita ser falso; seja relevante e claro. 
Assim, tendo em vista que a conversação é regida pelo Princípio de Cooperação e pelas Máximas, o ouvinte procura dar um sentido ao enunciado que esteja de acordo com essas implicações conversacionais. Para isso, as pessoas acreditam que seus interlocutores estão sendo o mais informativos, verdadeiros, relevantes e claros que conseguem. Esta crença interfere na compreensão, pois permite que muitas inferências desnecessárias sejam feitas. É possível que o falante não tenha certeza se os componentes da proposição são verdadeiros ou não. Essa incerteza é desconsiderada pelo ouvinte. Diante das tarefas lógicas, as pessoas podem assumir as informações disponibilizadas no problema como verdadeiras e relevantes para a resolução da tarefa, sem se preocupar em verificá-las e, por conseguinte, partindo de premissas incorretas chegar a conclusões erradas.

Embora o significado das partículas lógicas seja fornecido pelos seus esquemas de inferência básicos, ele pode ser ampliado pelas inferências "convidadas", que é o terceiro princípio. Geis e Zwicky (1971) verificaram que, em muitos casos, existe uma associação quase regular entre a forma lógica da sentença e a forma da inferência que ela "convida". Eles identificaram que, apesar de as sentenças com o ou poderem indicar tanto inclusão quanto exclusão, a interpretação geralmente favorecida é a exclusiva, como no seguinte exemplo: "André vai tocar violão ou jogar capoeira."

No entanto, quando a cláusula antecedente é um condicional, o ou é normalmente compreendido como inclusivo; como se pode observar a seguir:

(a) Se André tocar violão ou jogar capoeira, eu vou recitar um poema;

(b) Se André tocar violão e jogar capoeira, eu vou recitar um poema;

(c) Se André tocar violão ou jogar capoeira, eu vou recitar um poema. Mas, se ele fizer os dois, eu não farei nada, onde (a) sugere (b), mas não implica essa interpretação, pois (c) também é aceitável. Assim, como princípio geral, eles propõem que a sentença na forma $(X$ ou $Y) \rightarrow Z$ convida à inferência $(X$ e $Y) \rightarrow Z$.

Esses autores também verificaram que sentenças que expressam seqüência temporal convidam à inferência de que a primeira situação é a causa ou razão da segunda situação. Observe o seguinte exemplo: "Marta observou as crianças brincando e sorriu com prazer". Geralmente se infere que observar as crianças brincando foi o motivo que fez Marta sorrir com prazer. No entanto, entre essas sentenças há apenas uma relação de sugestão, e não de implicação.

Em suma, embora a TLM se volte exclusivamente para as inferências lógicas, ela não só admite a coexistência pacífica entre os diversos tipos de inferência, como também destaca a influência dos fatores pragmáticos sobre o raciocínio. Sua predição mais básica é que as pessoas realizam rotineiramente as inferências que dependem da aplicação das RRD; no entanto, as inferências que requerem estratégias mais complexas, que vão além das $R R D$, são realizadas em menor freqüência.

Com relação ao que propõem as teorias "concorrentes", é importante enfatizar que, apesar da TLM proclamar a existência de uma lógica mental universal inerente ao raciocínio humano, esta teoria destaca que a aplicação dos esquemas de inferência é limitada por princípios pragmáticos. Estes são usados para interpretar o significado do termo lógico no contexto em que está inserido. Desta forma, a teoria não desconsidera a influência do conteúdo sobre o raciocínio; mas acredita que o raciocínio humano não é apenas dependente do conteúdo, como afirmam Cheng e Holyoak (1985).

Os proponentes da TLM também não discordam de que as pessoas, ao raciocinar, elaboram modelos mentais. Entretanto, acreditam que não se pode explicar todo o raciocínio dedutivo recorrendo apenas à construção de 
modelos mentais, como afirma Johnson-Laird (1980).

Percebe-se, assim, que, apesar de ser possível detectar pontos convergentes entre estes modelos teóricos, as divergências ainda permanecem e precisam ser cada vez mais exploradas em futuras investigações. Assim, os estudiosos que tentam compreender e explicar o raciocínio dedutivo têm um longo caminho a percorrer, pois esta é uma área em que ainda há muito a ser "desvendado".

\section{REFERÊNCIAS BIBLIOGRÁFICAS}

BYRNE, R. M. J., Espino, O. \& Santamaria, C. (1999). Counterexamples and the suppression of inferences. Journal of Memory and Language, 40, 347-373.

BRAINE, M. D. S. (1978).On the relation between the natural logic of reasoning and the standard logic. Psychological Review, 85, 1-21.

BRAINE, M. D. S. \& O'Brien, D. P. (1998a). How to investigate mental logic and the syntax of thought. Em M. D. S. Braine \& D. P. O'Brien (Eds), Mental logic (pp.45-61). New Jersey: Lawrence Erlbaum Associates, Publishers.

BRAINE, M. D. S.\& O'Brien, D. P. (1998b). A theory of if: A lexical entry, reasoning program, and pragmatic principles. Em M. D. S. Braine \& D. P. O'Brien (Eds), Mental logic (pp. 199-244). New Jersey: Lawrence Erlbaum Associates, Publishers.

BRAINE, M. D. S.\& O'Brien, D. P. (1998c). The theory of mental-propositional logic: Description and illustration. Em M. D. S. Braine \& D. P. O'Brien (Eds), Mental logic (pp. 79-90). New Jersey: Lawrence Erlbaum Associates, Publishers.

BRAINE, M. D. S.; O'Brien, D. P., Noveck, I. A.; Samuels, M. C.; Lea, R. B.; Fisch, S. M. \& Yang, Y. (1998). Further evidence for the Theory: Predicting intermediate and multiple conclusions in propositional logic inference problems. Em M. D. S. Braine \& D. P. O'Brien
(Eds), Mental logic (pp. 145 - 198). New Jersey: Lawrence Erlbaum Associates, Publishers.

BRAINE, M. D. S.; Reiser, B.J. \& Rumain, B. (1998). Evidence for the Theory: Predicting the difficulty of propositional logic inference problems. Em M. D. S. Braine \& D. P. O'Brien (Eds), Mental logic (pp. 91-144). New Jersey: Lawrence Erlbaum Associates, Publishers.

CHENG, P. W. \& Holyoak, K. J. (1985). Pragmatic reasoning schemas. Cognitive Psychology, 17, 391-416.

DIAS, M. G. B. B. (1996). O desenvolvimento do raciocínio dedutivo. Em M. G. B. B. Dias \& A. G. Spinillo (Orgs.), Tópicos em psicologia cognitiva (pp.11-44). Recife: Editora Universitária da UFPE.

GEIS, M. L. \& Zwicky, A. M. (1971). On invited inferences. Linguistic Inquiry, 2, 561-566.

GRIGGS, R. A \& Cox, J. R. (1982). The elusive thematic-materials effect in Wason's selection task. British Journal of Psychology, 73, 407-420.

GUIMARÃES, E. (1995). Os limites do sentido: um estudo histórico e enunciativo da linguagem. Campinas: Pontes.

HOLYOAK, K. J. \& Cheng, P. W. (1995) Pragmatic reasoning from multiple points of view: A response. Thinking and Reasoning, 1, 373-388.

JACKSON, S. L. \& Griggs, R. A. (1990). The elusive pragmatic reasoning schema effect. Quarterly Journal of Experimental Psychology, $42,353-373$

JONHSON-LAIRD, P. N. (1980). Mental models in cognitive science. Cognitive Science, 4, 71-115.

JONHSON-LAIRD, P. N. \& Byrne, R. M. J. (1993). Mental models or formal rules? Behavioural and Brain Sciences, 16, 368-380.

JONHSON-LAIRD, P. N., Byrne, R. M. J. \& Shaeken, W. (1992). Propositional reasoning by model. Psychological Review, 99, 418-439. 
LEA, R. B. (1998). Logical inferences and comprehension: How mental-logic and text processing theories need each other. Em M. D. S. Braine \& D. P. O'Brien (Eds), Mental logic (pp. 63-78). New Jersey: Lawrence Erlbaum Associates, Publishers.

LEVINSON, S. C. (1983). Pragmatics. Cambridge: Cambridge University Press.

MARKOVITS, H. (1988). Conditional reasoning, representation, and empirical evidence on a concrete task. The Quarterly Journal of Experimental Psychology, 40 (3), 483-495.

O'BRIEN, D. P. (1995). Finding logic in human reasoning requires looking in the right places. Em: Newstead, S.E. \& Evans, J. St. B.T. (Eds.), Perspectives on Thinking and Reasoning. New Jersey: Lawrence Erlbaum Associates, Publishers.

O'BRIEN, D. P. (1998a). Introduction: Some background to the mental-logic theory and to the book. Em M. D. S. Braine \& D. P. O'Brien (Eds.), Mental logic (pp.1-6). New Jersey: Lawrence Erlbaum Associates, Publishers.

O'BRIEN, D. P. (1998b). Mental logic and irrationality: We can put a man on the moon, so why can't we solve those logical reasoning problems? Em M. D. S. Braine \& D. P. O'Brien (Eds), Mental logic (pp.23-43). New Jersey: Lawrence Erlbaum Associates, Publishers.

O'BRIEN, D. P.; Braine, M. D. S. \& Yang, Y. (1994). Propositional reasoning by mental models? Simple to refute in principle and in practice. Psychological Review, 101, 711-724.

VADEBONCOEUR, I. \& Markovits, H. (1999). The effect of instructions and information retrieval on accepting the premises in a conditional reasoning task. Thinking and Reasoning, 5(2), 97-113.

WASON, P. C. (1966). Reasoning. Em B. M. Foss (Ed.), New horizons in psychology. 1, pp.135-151. Harmondsworth: Penguin.

WASON, P. C. \& Johnson-Laird, P. N. (1972). Psychology of reasoning: Structure and content. Cambrigde, MA: Harvard University Press.

Recebido em 20 de agosto de 2001 e aceito em 30 de setembro de 2003. 
